\title{
BOOLEAN FUNCTIONS WITH SMALL SPECTRAL NORM
}

\author{
BEN GREEN AND TOM SANDERS
}

\begin{abstract}
Let $f: \mathbb{F}_{2}^{n} \rightarrow\{0,1\}$ be a boolean function, and suppose that the spectral norm $\|f\|_{A}:=\sum_{r}|\widehat{f}(r)|$ of $f$ is at most $M$. Then

$$
f=\sum_{j=1}^{L} \pm 1_{H_{j}}
$$

where $L \leqslant 2^{2^{C M^{4}}}$ and each $H_{j}$ is a subgroup of $\mathbb{F}_{2}^{n}$.

This result may be regarded as a quantitative analogue of the Cohen-Helson-Rudin structure theorem for idempotent measures in locally compact abelian groups.
\end{abstract}

\section{INTRODUCTION}

Let $G=\mathbb{F}_{2}^{n}$ be the $n$-dimensional cube, and let $f: G \rightarrow\{0,1\}$ be a boolean function, or more generally a function from $G$ to $\mathbb{R}$. In many works, particularly in theoretical computer science, the Fourier transform

$$
\widehat{f}(r):=\mathbb{E}_{x \in G} f(x)(-1)^{r^{T} x}=\frac{1}{|G|} \sum_{x \in G} f(x)(-1)^{r^{T} x}
$$

is considered. Here, $r$ lies in the dual group $\widehat{G}$ which we have identified with $G$ by choosing the scalar product $u^{T} v$.

It is natural to consider the $\ell^{p}$-norms

$$
\|\widehat{f}\|_{p}:=\left(\sum_{r \in \widehat{G}}|\widehat{f}(r)|^{p}\right)^{1 / p}
$$

for $1 \leqslant p<\infty$, as well as the $\ell^{\infty}$-norm $\|\widehat{f}\|_{\infty}:=\sup _{r}|\widehat{f}(r)|$.

There are many tools available for analysing these norms when $p \geqslant 2$, particularly when $p$ is $\infty$ or an even integer. When $1 \leqslant p<2$, however, the situation is in many ways rather mysterious. Of these cases, a very natural one is the endpoint $p=1$. In this case the norm $\|\widehat{f}\|_{1}$ is called the algebra norm, Wiener norm or spectral norm; we shall denote it by $\|f\|_{A}$. It is quite easy to show, using an instance of Young's inequality for convolutions, that

$$
\|f g\|_{A} \leqslant\|f\|_{A}\|g\|_{A}
$$

for any two functions $f, g: G \rightarrow \mathbb{R}$. This explains the term algebra norm.

The first author is a Clay Research Fellow, and thanks the Clay Mathematics Institute for their support. Much of this work was conducted while the second author was on a CMI-funded visit to Boston, and he thanks the first author for arranging this and the CMI for its support. Both authors would also like to thank the Massachusetts Institute of Technology for their hospitality. 
A basic question is the following.

Question 1.1. Let $M$ be a fixed positive real number and let $f: G \rightarrow\{0,1\}$ be a boolean function. When is $\|f\|_{A} \leqslant M$ ?

A partial answer to this question will be the main business of this paper. By far the most important feature of the problem is that we are asking it for boolean functions, which take only the values 0 or 1 . There is a ready supply of functions $f$ with $\|f\|_{A}$ small: Take for instance any pair $g, h: G \rightarrow \mathbb{R}$. Then $f:=g * h$ has

$$
\|f\|_{A}=\|g * h\|_{A}=\|\widehat{g} \widehat{h}\|_{1} \leqslant\|\widehat{g}\|_{2}\|\widehat{h}\|_{2}=\|g\|_{2}\|h\|_{2},
$$

which is small if $g$ and $h$ are small in $L^{2}$. It is rather hard, however, to construct a large supply of such functions which take only the values 0 and 1 .

To get a feel for the question, we prove a simple folklore result concerning the case $M=1$. In fact, by choosing a suitable argument from among the many available, one can cover the case $M<3 / 2$.

Proposition 1.2 (Boolean functions with tiny spectral norm). Let $f: G \rightarrow\{0,1\}$ be $a$ boolean function which does not vanish identically. Then either $f=1_{t+H}$, where $t+H$ is a coset of a subgroup of $G$, in which case $\|f\|_{A}=1$, or else $\|f\|_{A} \geqslant 3 / 2$.

Proof. First note that since $f$ is not identically zero we have $\|f\|_{\infty} \geqslant 1$, and so $\|f\|_{A} \geqslant$ $\|f\|_{\infty} \geqslant 1$, by the simplest instance of the Hausdorff-Young inequality.

Now suppose that $H \leqslant G$. The Fourier transform of $1_{t+H}$ is supported on $H^{\perp}:=\{r \in$ $\widehat{G}: r^{T} x=0$ for all $\left.x \in H\right\}$, and it has modulus $\left\|1_{H}\right\|_{1}$ there. It follows from this and the fact that $|H|\left|H^{\perp}\right|=|G|$ that $\|f\|_{A}=1$ when $f=1_{t+H}$.

To get the stronger statement claimed, we note that if $f$ is not (the characteristic function of ) a coset of a subgroup then there are four distinct points $x, x+h, x+k, x+$ $h+k$ forming a parallelogram in $G$ such that $f(x)=f(x+h)=f(x+k)=1$ but $f(x+h+k)=0$ (this is actually an if and only if statement - we leave the proof of both directions to the reader). Let

$$
\phi:=\delta_{x}+\delta_{x+h}+\delta_{x+k}-\delta_{x+h+k},
$$

thus $\phi(x)=\phi(x+h)=\phi(x+k)=|G|, \phi(x+h+k)=-|G|$ and $\phi(y)=0$ for all other $y$. Now we can compute that

$$
\langle f, \phi\rangle:=\mathbb{E}_{x \in G} f(x) \phi(x)=3 \text { and }\|\widehat{\phi}\|_{\infty}=2,
$$

and so it follows from Plancherel's theorem that

$$
3=\langle f, \phi\rangle=\langle\widehat{f}, \widehat{\phi}\rangle \leqslant\|\widehat{f}\|_{1}\|\widehat{\phi}\|_{\infty} \leqslant 2\|f\|_{A},
$$

which proves the result.

Remarks. We leave it to the reader to confirm that the constant $3 / 2$ is best possible. The result (and proof) are inspired by two papers of Saeki [Sae68a, Sae68b] in which the same question is addressed over all locally compact abelian groups $G$. In that more general setting the constant $3 / 2$ should be reduced to $\frac{1}{2}(1+\sqrt{2})$, and equality can occur in any group $G$ with an element of order 4 . 
Returning to our main question, let us recall that $\|\cdot\|_{A}$ is an algebra norm. Thus if $f_{1}, f_{2}: G \rightarrow\{0,1\}$ are functions for which $\left\|f_{1}\right\|_{A}$ and $\left\|f_{2}\right\|_{A}$ are small then the functions $f_{1} \vee f_{2}, f_{1} \wedge f_{2}, 1-f_{1}$ and $1-f_{2}$ also have this property. Loosely speaking, we refer to functions which can be obtained by a small number of operations of this kind from the basic functions $1_{t+H}$ as belonging to the coset ring of $G$. In fact, it is easy to see (ignoring quantitative issues for the time being) that all elements of the coset ring are in fact of the form

$$
\sum_{j=1}^{L} \pm 1_{H_{j}},
$$

for subgroups $H_{j} \leqslant G$ and some "small" $L$.

One trivially has the bound

$$
\left\|\sum_{j=1}^{L} \pm 1_{H_{j}}\right\|_{A} \leqslant L,
$$

and so it is rather natural to ask whether something like the converse is true; this is the main result of our paper.

Theorem 1.3 (Main theorem). Suppose that $f: G \rightarrow\{0,1\}$ has $\|f\|_{A} \leqslant M$. Then we may write

$$
f=\sum_{j=1}^{L} \pm 1_{H_{j}},
$$

where the $H_{j}$ are subgroups of $G$ and $L \leqslant 2^{2^{C M^{4}}}$ for some absolute constant $C$.

Remarks. The bound may seem unimpressive, and indeed in a sense it is. However it depends only on $M$, a feature which we believe is new to this paper. We do not dare to venture a guess as to the correct bound, and it seems to us that it would be difficult to use our method to reduce the number of exponentials below two. It may be possible to reduce the power 4 somewhat, although we have not attempted to do this.

The reader may wonder why we bothered to introduce the coset ring at all, when only the very natural functions (1.1) are involved in our theorem. The answer is that the description of the coset ring in the form (1.1) is specific to the case $G=\mathbb{F}_{2}^{n}$, and the phenomenon described by Theorem 1.3 is, in a sense, more general.

Indeed our entire approach was motivated by Cohen's celebrated idempotent theorem Coh60. Suppose that $G$ is a locally compact abelian group, and that $M(G)$ is the Banach algebra of finite measures on $G$ under convolution (see [Rud90, Appendix E] for details). We say that a measure $\mu \in M(G)$ is idempotent if $\mu * \mu=\mu$. Cohen's theorem is that $\mu$ is idempotent if any only if $\widehat{\mu}$ lies in the coset ring of $\Gamma=\widehat{G}$.

In our setting, Cohen's result implies that if $f: G \rightarrow\{0,1\}$ has $\|f\|_{A}<\infty$ then there is a decomposition of the form of (1.1) with $L$ finite. This is, of course, a vacuous result. It was, however, natural to start with Cohen's argument (as described in Rudin [Rud90]) and try to make it effective. A naïve attempt along these lines fails at several points and there are even "softer" proofs of Cohen's theorem that we have not managed to interpret 
in a finite setting at all, cf. [HMP86]. Nevertheless access to these classical results was crucial to our understanding and we could not have written this paper without them.

We also import some "modern" ingredients from additive combinatorics such as the Balog-Szemerédi theorem and Ruzsa's analogue of Freiman's theorem. It seems to the authors that it may be worth revisiting a number of classical results in the light of these developments.

It is possible that our methods, in combination with the ideas in Coh60, could lead to a fully quantitative proof of Cohen's idempotent theorem. We intend to pursue this direction in future work.

We conclude the introduction by remarking that the spectral norm of boolean functions is discussed in the computer science literature, but not in a great deal of detail. The papers [Bel92, Man94] show that functions which can be computed using a small binary decision tree have small spectral norm. Such functions are, however, rather special elements of the coset ring.

\section{NotATiOn}

Much of the notation we will use is implicit in the introduction, but it may be helpful to clarify things here. When working with functions on $G$, we will always use Haar probability measure. Integration with respect to this measure will be denoted by $\mathbb{E}_{x \in G}$, or sometimes just $\mathbb{E}$. If $f: G \rightarrow \mathbb{R}$ is a function and $1 \leqslant p<\infty$ then we define

$$
\|f\|_{p}:=\left(\mathbb{E}_{x \in G}|f(x)|^{p}\right)^{1 / p} .
$$

We also define $\|f\|_{\infty}:=\sup _{x}|f(x)|$ as usual. If $f_{1}, f_{2}: G \rightarrow \mathbb{R}$ are two functions then we set

$$
\left\langle f_{1}, f_{2}\right\rangle:=\mathbb{E}_{x \in G} f_{1}(x) f_{2}(x)
$$

and

$$
f_{1} * f_{2}(x):=\mathbb{E}_{y \in G} f_{1}(x) f_{2}(x-y) .
$$

When working with the Fourier transforms of functions we will use counting measure. Integration with respect to this measure will be denoted by $\sum$ as usual. We defined the $\ell^{p}$ norms in the introduction. Note also Plancherel's identity, which implies that

$$
\left\langle f_{1}, f_{2}\right\rangle=\left\langle\widehat{f}_{1}, \widehat{f}_{2}\right\rangle:=\sum_{r} \widehat{f}_{1}(r) \widehat{f}_{2}(r)
$$

We will occasionally write, e.g., $E^{\wedge}$ when taking the Fourier transform of a particularly complicated expression $E$.

Finally, a word concerning absolute constants. The letter $C$ will always denote an absolute constant, but the exact value of this constant may change form expression to expression. If in doubt, the reader should recall that all instances of $C$ could, if desired, be replaced by specific constants in such a way that all our proofs are correct. 


\section{Almost integer-VAlued functions and almost homomorphisms}

A key feature of this paper is that we cannot work entirely within the "category" of boolean functions. We must also consider more general functions which are close to being integer-valued.

Definition 3.1 (Almost integer-valued functions). Let $\epsilon \in(0,1 / 2)$. We say that a function $f: G \rightarrow \mathbb{R}$ is $\epsilon$-almost integer-valued if there is a function $f_{\mathbb{Z}}: G \rightarrow \mathbb{Z}$ such that $\left\|f-f_{\mathbb{Z}}\right\|_{\infty} \leqslant \epsilon$.

We will need to study the behaviour of almost integer-valued functions under a certain class of map. Let $H$ be a subgroup of $G$. For any function $f: G \rightarrow \mathbb{R}$, we define $\psi_{H} f$ by

$$
\left(\psi_{H} f\right)(x):=\mathbb{E}_{y \in x+H} f(y)=f * \mu_{H}(x),
$$

where $\mu_{H}$ denotes the Haar probability measure on $H$. Equivalently, one may define $\psi_{H}$ in terms of its Fourier transform by

$$
\left(\psi_{H} f\right)^{\wedge}(r):=\widehat{f}(r) 1_{H^{\perp}}(r),
$$

where the subgroup $H^{\perp} \leqslant \widehat{G}$ is the annihilator of $H$, defined by

$$
H^{\perp}:=\left\{r \in \widehat{G}: r^{T} x=0 \text { for all } x \in H\right\} .
$$

The following simple properties of $\psi_{H}$ follow immediately from the above definitions.

Lemma 3.2 (Simple properties of $\psi_{H}$ ). The norm of $\psi_{H}$ is at most 1 in both the operator norm induced by the spectral norm and in that induced by the $L^{\infty}$-norm. That is to say

$$
\left\|\psi_{H} f\right\|_{A} \leqslant\|f\|_{A} \text { and }\left\|\psi_{H} f\right\|_{\infty} \leqslant\|f\|_{\infty} \text { for all } f: G \rightarrow \mathbb{R} \text {. }
$$

Definition 3.3 (Spectral support). Let $\eta>0$ be a parameter, let $f: G \rightarrow \mathbb{R}$ be a function, and suppose that $H \leqslant G$. Then we say that $f$ is $\eta$-spectrally supported on $H$ if

$$
\sup _{r \notin H^{\perp}} \sum_{r^{\prime} \in r+H^{\perp}}\left|\widehat{f}\left(r^{\prime}\right)\right| \leqslant \eta \text {. }
$$

Note that we do not assume that $\widehat{f}$ has substantial mass on $H^{\perp}$ itself.

Lemma 3.4 (Finding the spectral support). Let $H \leqslant G$ be any subgroup, let $\eta>0$ be any parameter, and let $f: G \rightarrow \mathbb{R}$ be a function with $\|f\|_{A} \leqslant M$. Then there is a subgroup $H^{\prime} \leqslant H$ with

$$
\operatorname{codim}\left(H: H^{\prime}\right) \leqslant M / \eta
$$

such that $f$ is $\eta$-spectrally supported on $H^{\prime}$.

Proof. Set $H_{0}:=H$. We define a descending sequence $H_{0} \geqslant H_{1} \geqslant \ldots$ of subgroups with $\operatorname{codim}\left(H_{i}: H_{i+1}\right)=1$.

If, at some stage, $f$ is $\eta$-spectrally supported on $H_{i}$ then we stop. If not, there is some $r_{i} \notin H_{i}^{\perp}$ such that

$$
\sum_{r^{\prime} \in r_{i}+H_{i}^{\perp}}\left|\widehat{f}\left(r^{\prime}\right)\right|>\eta .
$$


Define $H_{i+1}^{\perp}$ to be the subgroup of $G$ generated by $r_{i}$ and $H_{i}^{\perp}$. It is clear that for any $j$ we have

$$
\|f\|_{A} \geqslant \sum_{i=0}^{j} \sum_{r^{\prime} \in r_{i}+H_{i}^{\perp}}\left|\widehat{f}\left(r^{\prime}\right)\right|,
$$

and so this inductive process must terminate after no more than $M / \eta$ steps.

The purpose of Definition 3.3 and Lemma 3.4 is to allow us to use the following approximate homomorphism property.

Lemma $3.5\left(\psi_{H}\right.$ is an approximate homomorphism). Suppose that $f, g: G \rightarrow \mathbb{R}$ are two functions, and that $f$ is $\eta$-spectrally supported on $H$. Then

$$
\left\|\psi_{H}(f g)-\psi_{H}(f) \psi_{H}(g)\right\|_{A} \leqslant \eta\|g\|_{A} .
$$

Proof. We have

$$
\left\|\psi_{H}(f g)-\psi_{H}(f) \psi_{H}(g)\right\|_{A}=\sum_{r}\left|\widehat{f} * \widehat{g}(r) 1_{H^{\perp}}(r)-\widehat{f} 1_{H^{\perp}} * \widehat{g} 1_{H^{\perp}}(r)\right| .
$$

However

$$
\begin{aligned}
\widehat{f} * \widehat{g}(r) 1_{H^{\perp}}(r) & =\sum_{s} \widehat{f}(r-s) \widehat{g}(s) 1_{H^{\perp}}(r) \\
& =\sum_{s} \widehat{f}(r-s) \widehat{g}(s) 1_{H^{\perp}}(r-s) 1_{H^{\perp}}(s)+\sum_{s \notin H^{\perp}} \widehat{f}(r-s) \widehat{g}(s) 1_{H^{\perp}}(r) \\
& =\widehat{f} 1_{H^{\perp}} * \widehat{g} 1_{H^{\perp}}(r)+\sum_{s \notin H^{\perp}} \widehat{f}(r-s) \widehat{g}(s) 1_{H^{\perp}}(r) .
\end{aligned}
$$

Now simply note that

$$
\begin{aligned}
\sum_{r}\left|\sum_{s \notin H^{\perp}} \widehat{f}(r-s) \widehat{g}(s) 1_{H^{\perp}}(r)\right| & \leqslant \sum_{s \notin H^{\perp}} \sum_{r \in H^{\perp}}|\widehat{f}(r-s)||\widehat{g}(s)| \\
& \leqslant \sum_{s}|\widehat{g}(s)| \sup _{s \notin H^{\perp}} \sum_{r \in H^{\perp}}|\widehat{f}(r-s)| \\
& \leqslant \eta\|g\|_{A} .
\end{aligned}
$$

This completes the proof.

Our aim is to show that, provided the parameter $\eta$ is suitably small, the map $\psi_{H}$ preserves almost integer-valued functions.

Lemma 3.6. Let $d \geqslant 0$ be an integer, and write $P_{d}(X):=4^{d}(2 d) !^{-1} \prod_{j=-d}^{d}(X-j)$. Let $\epsilon, \delta \leqslant 1 / 2$ be positive real parameters. Let $f: G \rightarrow \mathbb{R}$ be a function.

(1) If $f$ is $\epsilon$-almost integer-valued and $\|f\|_{\infty} \leqslant d$ then $\left\|P_{d}(f)\right\|_{\infty} \leqslant \epsilon 4^{d}$.

(2) If $\left\|P_{d}(f)\right\|_{\infty} \leqslant \delta$ then $f$ is $\delta$-almost integer-valued.

Proof. To prove the first statement, simply note that

$$
\left\|P_{d}(f)\right\|_{\infty} \leqslant 4^{d}(2 d) !^{-1} \cdot(2 d) ! \sup _{x} \inf _{j}|f(x)-j| \leqslant \epsilon 4^{d} .
$$


To prove the second, observe that

$$
\left|P_{d}(t)\right| \geqslant\left|P_{d}(\bar{t})\right|
$$

for all $t \in \mathbb{R}$, where $\bar{t} \equiv t(\bmod 1)$ and lies in the interval $(-1 / 2,1 / 2]$. Furthermore one may easily confirm that

$$
\left|P_{d}(\bar{t})\right| \geqslant \frac{|\bar{t}| \cdot 4^{d} d ! \prod_{j=0}^{d-1}\left(j+\frac{1}{2}\right)}{(2 d) !} \geqslant|\bar{t}|
$$

for all $t$. It follows that for all $x$ the distance from $f(x)$ to the nearest integer is no more than $\delta$.

Remark. We have normalised the polynomials $P_{d}$ slightly arbitrarily, so that no factors were lost in (2). This makes no essential difference to the argument.

To apply this, we combine it with the rest of the results of this section to obtain the following corollary.

Proposition $3.7\left(\psi_{H}\right.$ preserves almost integer-valued functions). Suppose that $f$ : $G \rightarrow \mathbb{R}$ is $\epsilon$-almost integer-valued, and that $\|f\|_{A} \leqslant M$ for some $M \geqslant 1 / 2$. Suppose that $\eta \leqslant 2^{-C M(1+\log M)} \epsilon$ for some suitably large $C$ and that $f$ is $\eta$-spectrally supported on $H$. Then both $\psi_{H} f$ and $f-\psi_{H} f$ are $\left(2^{C M} \epsilon\right)$-almost integer-valued.

Proof. It clearly suffices to prove the result for $\psi_{H} f$, as the sum or difference of two almost integer-valued functions is almost integer-valued. Set $d:=\lceil M\rceil$. Since $\|f\|_{\infty} \leqslant$ $\|f\|_{A} \leqslant M$, Lemma 3.6 (1) implies that

$$
\left\|P_{d}(f)\right\|_{\infty} \leqslant 2^{C M} \epsilon .
$$

From Lemma 3.2 it follows that

$$
\left\|\psi_{H}\left(P_{d}(f)\right)\right\|_{\infty} \leqslant 2^{C M} \epsilon .
$$

Now an easy induction based on Lemma 3.5 (and Lemma 3.2) confirms that

$$
\left\|\psi_{H}\left(f^{n}\right)-\left(\psi_{H} f\right)^{n}\right\|_{A} \leqslant \eta(n-1) M^{n-1},
$$

for any positive integer $n$. It follows from this that

$$
\left\|\psi_{H}\left(P_{d}(f)\right)-P_{d}\left(\psi_{H} f\right)\right\|_{A} \leqslant 2^{C M(1+\log M)} \eta \leqslant 2^{C M} \epsilon,
$$

and hence in view of (3.1) that

$$
\left\|P_{d}\left(\psi_{H} f\right)\right\|_{\infty} \leqslant 2^{C M} \epsilon
$$

The result is now an immediate consequence of Lemma 3.6 (2).

\section{Ruzsa's analogue of Freiman's theorem}

In the next two sections we use variants of a well-known sequence of arguments in additive combinatorics. The objective is to prove Proposition 5.1, which roughly speaking states that a function with small $A(G)$-norm concentrates on a subspace. We will supply original references for the results we use, but would also recommend the book [TV06] as a general resource for this subject. 
When we actually prove Proposition 5.1 we will find ourselves dealing with a set $A \subseteq G$ with small doubling, that is to say a set $A$ with $\mathbb{E} 1_{A+A} \leqslant K \mathbb{E} 1_{A}$ for some "not too large" $K$. There is a beautiful theorem of Imre Ruzsa [Ruz99] (see also [San08]) which states that in this case $A$ is contained in a subgroup $H \leqslant G$ with density at most $K^{2} 2^{K^{4}} \mathbb{E} 1_{A}$. This certainly implies that

$$
\mathbb{E}_{x \in A} 1_{H}(x) \geqslant 1 \text { and } \mathbb{E}_{x \in H} 1_{A}(x) \geqslant 2^{-C K^{C}} .
$$

One could use this result as it is, and obtain a bound in Theorem 1.3 with a three-fold iterated exponential. To reduce the number of exponentials to two, we need a different version of Ruzsa's result, in which we shall replace (4.1) with

$$
\mathbb{E}_{x \in A} 1_{H}(x) \geqslant 2^{-C K^{C}} \text { and } \mathbb{E}_{x \in H} 1_{A}(x) \geqslant c K^{-C} .
$$

A more precise version of the following proposition, which is the main result of the section, will be contained in a forthcoming paper of the first author and Terence Tao. The authors are grateful to the latter for useful discussions regarding this circle of ideas.

Proposition 4.1 (Freiman in torsion groups, refined). Suppose that $A \subseteq G$ is a set with $\mathbb{E} 1_{A+A} \leqslant K \mathbb{E} 1_{A}$. Then there is a subgroup $H \leqslant G$ such that

$$
\mathbb{E}_{x \in A} 1_{H}(x) \geqslant 2^{-C K^{C}} \text { and } \mathbb{E}_{x \in H} 1_{A}(x) \geqslant c K^{-C} .
$$

Remark. It is an important unsolved problem to decide whether or not one may replace $2^{-C K^{C}}$ by a polynomial in the first bound. This is known as the Polynomial FreimanRuzsa conjecture (PFR); see for example Gre05]. The truth of this conjecture, however, would not make an essential difference to the bound we obtain in Theorem 1.3 .

To prove Proposition 4.1 we need to set up a little notation. Write $\alpha:=\mathbb{E} 1_{A}$ for the density of $A$ in $G$. Put

$$
\nu^{(4)}(x):=1_{A} * 1_{A} * 1_{A} * 1_{A}(x),
$$

and for any parameter $\eta>0$ define

$$
S_{\eta}:=\left\{x \in G: \nu^{(4)}(x) \geqslant \eta \alpha^{3}\right\} .
$$

For a parameter $\rho \in(0,1)$, we write (as is becoming standard)

$$
\operatorname{Spec}_{\rho}(A):=\left\{r \in \widehat{G}:\left|\widehat{1}_{A}(r)\right| \geqslant \rho \alpha\right\} .
$$

We begin by recording a well-known argument of Bogolyubov [Bog39] in this language.

Lemma 4.2 (Bogolyubov's argument). Suppose that $A \subset G$. Let $\delta, \epsilon \in(0,1)$ be any parameters and set $\rho:=(\epsilon / 2)^{1 / 2}$ and $H:=\operatorname{Spec}_{\rho}(A)^{\perp}$. Then

$$
S_{\delta}+H \subseteq S_{\delta-\epsilon} .
$$

Proof. Suppose that $x \in S_{\delta}$ and that $h \in H$. Then we have

$$
\begin{aligned}
\nu^{(4)}(x+h) & =\sum_{r \in \widehat{G}}\left|\widehat{1}_{A}(r)\right|^{4}(-1)^{r^{T}(x+h)} \\
& =\sum_{r \in \operatorname{Spec}_{\rho}(A)}\left|\widehat{1}_{A}(r)\right|^{4}(-1)^{r^{T} x}+\sum_{r \notin \operatorname{Spec}_{\rho}(A)}\left|\widehat{1}_{A}(r)\right|^{4}(-1)^{r^{T}(x+h)} \\
& \geqslant \nu^{(4)}(x)-2 \sum_{r \notin \operatorname{Spec}_{\rho}(A)}\left|\widehat{1}_{A}(r)\right|^{4} .
\end{aligned}
$$


Thus we only need observe, using Parseval's identity and the definition of $\operatorname{Spec}_{\rho}(A)$, that

$$
\sum_{r \notin \operatorname{Spec}_{\rho}(A)}\left|\widehat{1}_{A}(r)\right|^{4} \leqslant \sup _{r \notin \operatorname{Spec}_{\rho}(A)}\left|\widehat{1}_{A}(r)\right|^{2} \sum_{r \in G}\left|\widehat{1}_{A}(r)\right|^{2} \leqslant(\rho \alpha)^{2} \cdot \alpha=\epsilon \alpha^{3} / 2 .
$$

The next two lemmas are the vehicles by which we leverage the assumption that $A$ has small doubling. The first states that $A$ has large density on a translate of $S_{\eta}$, provided $\eta$ is sufficiently small.

Lemma 4.3. Suppose that $A \subseteq G$, that $\mathbb{E} 1_{A+A} \leqslant K \mathbb{E} 1_{A}$ and that $\eta \leqslant 1 / 2 K^{4}$ is a parameter. Then $\mathbb{E} 1_{S_{\eta}} \geqslant \alpha / 2$ and

$$
\left\|1_{A} * 1_{S_{\eta}}\right\|_{\infty} \geqslant \eta \alpha / 2 .
$$

Proof. Averaging $\nu^{(4)}$ over $x \in G$, we get

$$
\begin{aligned}
\alpha^{4} & =\mathbb{E} \nu^{(4)} \\
& \leqslant \alpha^{3} \mathbb{E} 1_{S_{\eta}}+\eta \alpha^{3} \mathbb{E} 1_{4 A} \\
& \leqslant \alpha^{3} \mathbb{E} 1_{S_{\eta}}+\eta \alpha^{4} K^{4} \\
& \leqslant \alpha^{3} \mathbb{E} 1_{S_{\eta}}+\alpha^{4} / 2,
\end{aligned}
$$

where the second inequality follows from the Plünnecke-Ruzsa inequalities [Ruz96], and the third from the condition on $\eta$. The first conclusion of the lemma follows immediately upon rearranging.

For the second part we use the first to see that

$$
\begin{aligned}
\eta \alpha^{4} / 2 & \leqslant \eta \alpha^{3} \mathbb{E} 1_{S_{\eta}} \\
& \leqslant \mathbb{E}\left(1_{S_{\eta}} \nu^{(4)}\right) \\
& =\left\langle 1_{S_{\eta}}, 1_{A} * 1_{A} * 1_{A} * 1_{A}\right\rangle \\
& =\left\langle 1_{A} * 1_{S_{\eta}}, 1_{A} * 1_{A} * 1_{A}\right\rangle \\
& \leqslant\left\|1_{A} * 1_{S_{\eta}}\right\|_{\infty} \mathbb{E} 1_{A} * 1_{A} * 1_{A} .
\end{aligned}
$$

The conclusion follows immediately since $\mathbb{E} 1_{A} * 1_{A} * 1_{A}=\alpha^{3}$.

Lemma 4.4. Suppose that $A \subseteq G$ has density $\alpha:=\mathbb{E} 1_{A}$ and that $\mathbb{E} 1_{A+A} \leqslant K \mathbb{E} 1_{A}$. Then there is a subgroup $H \leqslant G$ with $\mathbb{E} 1_{H} \geqslant(\alpha / 2)^{-C K^{12}}$ and some $\eta$ with $1 / 4 K^{4} \leqslant$ $\eta \leqslant 1 / 2 K^{4}$, such that $S_{\eta}$ is a union of cosets of $H$ together with an exceptional set $X$ satisfying $\mathbb{E} 1_{X} \leqslant \alpha / 16 K^{4}$.

Proof. Let $\eta_{0}:=1 / 2 K^{4}$ and set $\epsilon:=1 / 64 K^{12}$. Consider the nested sequence

$$
S_{\eta_{0}} \subseteq S_{\eta_{0}-\epsilon} \subseteq \cdots \subseteq S_{\eta_{0}-(L-1) \epsilon}
$$

where $L:=1 / 4 K^{4} \epsilon$. By the Plünnecke-Ruzsa inequalities we have Ruz96]

$$
\mathbb{E} 1_{S_{\eta}} \leqslant \mathbb{E} 1_{4 A} \leqslant K^{4} \alpha
$$


for any $\eta$, and therefore by the pigeonhole principle there is some $j$ with $0 \leqslant j<L$ such that

$$
\mathbb{E} 1_{S_{\eta_{0}-(j+1) \epsilon} \backslash S_{\eta_{0}-j \epsilon}} \leqslant \alpha / 16 K^{4} .
$$

Now we apply Lemma 4.2. Writing $H=\operatorname{Spec}_{\rho}(A)^{\perp}$ where $\rho:=1 / 16 K^{6}$, we know from that lemma that

$$
S_{\eta_{0}-j \epsilon}+H \subseteq S_{\eta_{0}-(j+1) \epsilon}
$$

Thus $S_{\eta_{0}-(j+1) \epsilon}$ can be written as a union of cosets of $H$ together with an exceptional set $X$ of density at most $\alpha / 16 K^{4}$.

It remains to establish the claimed lower bound on $\mathbb{E} 1_{H}$. By a lemma of Chang Cha02, Lemma 3.1] (see also [Gre02, Lecture 14, Lemma 3] and [Rud60, TV06]) the set $\operatorname{Spec}_{\rho}(A)$ is contained in a subgroup of $G$ with dimension $O\left(\rho^{-2}(1+\log (1 / \alpha))\right)=O\left(K^{12} \log \alpha^{-1}\right)$. This concludes the proof.

Proof of Proposition 4.1. It is sufficient to prove the proposition when $G=\langle A\rangle$ in which case, by Ruzsa's Theorem [Ruz99], we have $\alpha \geqslant K^{-2} 2^{-K^{4}}$.

Apply Lemma 4.4 to get a subgroup $H^{\prime}$ with $\mathbb{E} 1_{H^{\prime}} \geqslant 2^{-C K^{16}}$ and some $\eta$ with $1 / 4 K^{4} \leqslant$ $\eta \leqslant 1 / 2 K^{4}$, such that

$$
S_{\eta}=X \cup \bigcup_{y \in Y}\left(y+H^{\prime}\right)
$$

where $\mathbb{E} 1_{X} \leqslant \alpha / 16 K^{4}$. Writing $\mu_{H^{\prime}}:=1_{H^{\prime}} / \mathbb{E} 1_{H^{\prime}}$ for the Haar measure on $H^{\prime}$, we have for all $x \in G$ that

$$
\begin{aligned}
1_{A} * 1_{S_{\eta}} * \mu_{H^{\prime}}(x) & \geqslant 1_{A} * 1_{S_{\eta} \backslash X} * \mu_{H^{\prime}}(x) \\
& =1_{A} * 1_{S_{\eta} \backslash X}(x) \\
& =1_{A} * 1_{S_{\eta}}(x)-1_{A} * 1_{X}(x) \\
& \geqslant 1_{A} * 1_{S_{\eta}}(x)-\mathbb{E} 1_{X} \\
& \geqslant 1_{A} * 1_{S_{\eta}}(x)-\alpha / 16 K^{4} .
\end{aligned}
$$

It follows from this, Lemma 4.3 and the assumption that $\eta \geqslant 1 / 4 K^{4}$ that

$$
\left\|1_{A} * 1_{S_{\eta}} * \mu_{H^{\prime}}\right\|_{\infty} \geqslant\left\|1_{A} * 1_{S_{\eta}}\right\|_{\infty}-\alpha / 16 K^{4} \geqslant \eta \alpha / 2-\alpha / 16 K^{4} \geqslant \alpha / 16 K^{4} .
$$

Furthermore by the Plünnecke-Ruzsa inequalities [Ruz96] we have

$$
\left\|1_{A} * 1_{S_{\eta}} * \mu_{H^{\prime}}\right\|_{\infty} \leqslant\left\|1_{A} * \mu_{H^{\prime}}\right\|_{\infty} \mathbb{E} 1_{S_{\eta}} \leqslant\left\|1_{A} * \mu_{H^{\prime}}\right\|_{\infty} \mathbb{E} 1_{4 A} \leqslant K^{4} \alpha\left\|1_{A} * \mu_{H^{\prime}}\right\|_{\infty} .
$$

Comparing with (4.2) leads immediately to

$$
\left\|1_{A} * \mu_{H^{\prime}}\right\|_{\infty} \geqslant 1 / 16 K^{8} .
$$

We have found a coset of $H^{\prime}$ on which the relative density of $A$ is at least $1 / 16 K^{8}$; by adjoining the zero element to $H^{\prime}$ if necessary, one obtains a subgroup $H$ on which the relative density of $A$ is at least $1 / 32 K^{8}$, that is to say

$$
\mathbb{E}_{x \in H} 1_{A}(x) \geqslant 1 / 32 K^{8} \text {. }
$$

To complete the proof of Proposition 4.1 it remains to note that

$$
\mathbb{E}_{x \in A} 1_{H}(x)=\frac{\mathbb{E} 1_{H}}{\mathbb{E} 1_{A}} \cdot \mathbb{E}_{x \in H} 1_{A}(x) \geqslant\left(\mathbb{E} 1_{H}\right) \mathbb{E}_{x \in H} 1_{A}(x) \geqslant 2^{-C K^{16}}
$$




\section{Concentration on a subgroup}

Proposition 5.1 (Concentration on a subgroup). Suppose that $f: G \rightarrow \mathbb{R}$ is an $\epsilon$ integer-valued function with $\|f\|_{A} \leqslant M$, where $M \geqslant 1 / 2$ and $\epsilon \leqslant 2^{-C M^{4}}$. Then there is a subgroup $H \leqslant G$ with

$$
\mathbb{E} 1_{H} \geqslant 2^{-2^{C M^{4}}}\left\|f_{\mathbb{Z}}\right\|_{1}
$$

and

$$
\sup _{x \in G}\left|\mathbb{E}_{y \in x+H} f(y)\right|=\left\|\psi_{H} f\right\|_{\infty} \geqslant 2^{-C M^{4}} .
$$

Definition 5.2 (Arithmetic connectedness). Let $m$ be a positive integer. Suppose that $A \subseteq G$ is a set with $0 \notin A$. Then we say that $A$ is $m$-arithmetically connected if, for any choice of distinct $a_{1}, \ldots, a_{m} \in A$, one of the following alternatives holds:

(1) The vectors $a_{1}, \ldots, a_{m}$ are linearly dependent;

(2) The vectors $a_{1}, \ldots, a_{m}$ are linearly independent but there exists a further $a^{\prime} \in A$ such that $a^{\prime}$ lies in the linear span of the $a_{i}$.

The next lemma imports the tools we developed in $\$ 4$. The result allows us to weaken the condition of small doubling in Proposition 4.1 to that of arithmetic connectedness.

Lemma 5.3. Suppose that $m$ is a positive integer and that $A \subseteq G$ is a set with $0 \notin A$. Suppose that $A$ is m-arithmetically connected. Then there is a subgroup $H \leqslant G$ such that

$$
\mathbb{E}_{x \in A} 1_{H}(x) \geqslant 2^{-2^{C m}} \text { and } \mathbb{E}_{x \in H} 1_{A}(x) \geqslant 2^{-C m} \text {. }
$$

Proof. If $|A|<m^{2}$ the result is trivial, so we stipulate that $|A| \geqslant m^{2}$. Pick any $m$-tuple $\left(a_{1}, \ldots, a_{m}\right)$ of distinct elements of $A$. With the stipulated lower bound on $|A|$, there are at least $|A|^{m} / 2$ such $m$-tuples. We know that either the vectors $a_{1}, \ldots, a_{m}$ are linearly dependent, or else there is a further $a^{\prime} \in A$ such that $a^{\prime}$ lies in the linear span of the $a_{i}$. In either situation there is some linear relation

$$
\lambda_{1} a_{1}+\cdots+\lambda_{m} a_{m}+\lambda^{\prime} a^{\prime}=0
$$

where $\vec{\lambda}:=\left(\lambda_{1}, \ldots, \lambda_{m}, \lambda^{\prime}\right)$ has elements in $\mathbb{F}_{2}$ and, since $0 \notin A$ and the $a_{i}$ s are distinct, at least three of the components of $\vec{\lambda}$ are nonzero. By the pigeonhole principle, it follows that there is some $\vec{\lambda}$ such that the linear equation

$$
\lambda_{1} x_{1}+\cdots+\lambda_{m} x_{m}+\lambda^{\prime} x^{\prime}=0
$$

has at least $|A|^{m} / 2^{m+2}$ solutions with $x_{1}, \ldots, x_{m}, x^{\prime} \in A$. Removing the zero coefficients, we may thus assert that there is some $r, 3 \leqslant r \leqslant m+1$, such that the equation

$$
x_{1}+\cdots+x_{r}=0
$$

has at least $|A|^{r-1} / 2^{m+2}$ solutions with $x_{1}, \ldots, x_{r} \in A$. Note that this is a strong structural statement about $A$, since the maximum possible number of solutions to such an equation is $|A|^{r-1}$.

We claim that there are at least $2^{-2 m-4}|A|^{3}$ solutions to $x_{1}+x_{2}=x_{3}+x_{4}$ with $x_{i} \in A$. To see this, write $R_{l}(x)$ for the number of $l$-tuples $\left(x_{1}, \ldots, x_{l}\right) \in A^{l}$ such that $x_{1}+\cdots+x_{l}=$ 
$x$, and note that

$$
\sum_{x} R_{2}(x) R_{r-2}(x)=R_{r}(0) \geqslant|A|^{r-1} / 2^{m+2} .
$$

Noting that $R_{r-2}(x) \leqslant|A|^{r-3}$ for all $x$ (here, of course, it is important that $r \geqslant 3$ ) we see from the Cauchy-Schwarz inequality that

$$
\sum_{x} R_{2}(x)^{2} \geqslant \frac{|A|^{2(r-1)}}{2^{2 m+4}\left(\sum_{x} R_{r-2}(x)^{2}\right)} \geqslant 2^{-2 m-4}|A|^{3},
$$

confirming the claim.

It now follows from the Balog-Szemerédi-Gowers theorem [Gow98, Proposition 12] that there is some set $A^{\prime} \subseteq A, \mathbb{E} 1_{A^{\prime}} \geqslant 2^{-C m} \mathbb{E} 1_{A}$, such that $\mathbb{E} 1_{A^{\prime}+A^{\prime}} \leqslant 2^{C m} \mathbb{E} 1_{A^{\prime}}$. By Proposition 4.1 there is a subgroup $H \leqslant G$ with

$$
\mathbb{E}_{x \in A^{\prime}} 1_{H}(x) \geqslant 2^{-2^{C m}} \text { and } \mathbb{E}_{x \in H} 1_{A^{\prime}}(x) \geqslant 2^{-C m} .
$$

The result follows since $\mathbb{E} 1_{A} \geqslant \mathbb{E} 1_{A^{\prime}} \geqslant 2^{-C m} \mathbb{E} 1_{A}$.

Proof of Proposition 5.1. We begin by decomposing $f^{2}$ as $g+h$ where $g=f_{\mathbb{Z}}^{2}$ and $h=f^{2}-g$. We have

$$
\|h\|_{\infty}=\left\|f^{2}-f_{\mathbb{Z}}^{2}\right\|_{\infty} \leqslant\left\|f-f_{\mathbb{Z}}\right\|_{\infty}\left\|f+f_{\mathbb{Z}}\right\|_{\infty} \leqslant 4 \epsilon M
$$

the latter inequality being a consequence of the fact that $\|f\|_{\infty} \leqslant\|f\|_{A} \leqslant M$ and that $\left\|f_{\mathbb{Z}}\right\|_{\infty} \leqslant\|f\|_{\infty}+\epsilon$.

Set $m=\left\lceil(2 M)^{4}\right\rceil$ and suppose that $V \leqslant G$ is a subgroup of dimension $m$. We have

$$
\left\|f^{2} 1_{V}\right\|_{A} \leqslant\|f\|_{A}^{2}\left\|1_{V}\right\|_{A}=\|f\|_{A}^{2} \leqslant M^{2} .
$$

In view of the trivial estimate

$$
\left\|h 1_{V}\right\|_{A} \leqslant \sum_{x \in V}\left\|h(x) 1_{x}\right\|_{A} \leqslant 2^{m}\|h\|_{\infty} \leqslant 2^{m+2} \epsilon M
$$

it follows from the triangle inequality and the assumption on $\epsilon$ that

$$
\left\|g 1_{V}\right\|_{A} \leqslant M^{2}+2^{m+2} \epsilon M \leqslant 2 M^{2} .
$$

Write $A:=\operatorname{Supp}(g)=\operatorname{Supp}\left(f_{\mathbb{Z}}\right)$. If $A$ is all of $G$ then the proposition follows trivially so we may assume that this is not the case. Hence by replacing $f(x)$ by $f(x+y)$ for some $y \notin A$, we may assume without loss of generality that $0 \notin A$. We claim that $A$ is $m$ arithmetically connected in the sense of Definition 5.2. If this is not the case then there are elements $a_{1}, \ldots, a_{m} \in A$ such that the vectors $a_{1}, \ldots, a_{m}$ are linearly independent, and such that there is no $a^{\prime} \in A$ with $a^{\prime}$ in the linear span of the $a_{i}$. Writing $V$ for the subgroup of $G$ spanned by the $a_{i}$, this means that the support of $g 1_{V}$ is precisely $\left\{a_{1}, \ldots, a_{m}\right\}$.

Thus we have

$$
g 1_{V}(x)=\sum_{i=1}^{m} g\left(a_{i}\right) 1_{a_{i}}(x)
$$


Now we may compute that

$$
\left\|\left(g 1_{V}\right)^{\wedge}\right\|_{2}^{2}=\left\|g 1_{V}\right\|_{2}^{2}=\frac{1}{|G|} \sum_{i=1}^{m}\left|g\left(a_{i}\right)\right|^{2} \geqslant \frac{m}{|G|}
$$

and that

$$
\left\|\left(g 1_{V}\right)^{\wedge}\right\|_{4}^{4}=\frac{1}{|G|^{3}} \sum_{\substack{i_{1}, i_{2}, i_{3}, i_{4} \\ a_{i_{1}}+a_{i_{2}}=a_{i_{3}}+a_{i_{4}}}}\left|g\left(a_{i}\right)\right|^{4} \leqslant \frac{3}{|G|^{3}}\left(\sum_{i=1}^{m}\left|g\left(a_{i}\right)\right|^{2}\right)^{2}=\frac{3}{|G|}\left\|\left(g 1_{V}\right)^{\wedge}\right\|_{2}^{4},
$$

the middle inequality following from the observation that $a_{i_{1}}+a_{i_{2}}=a_{i_{3}}+a_{i_{4}}$ only if $i_{1}=i_{2}$ and $i_{3}=i_{4}$, or $i_{1}=i_{3}$ and $i_{2}=i_{4}$, or $i_{1}=i_{4}$ and $i_{2}=i_{3}$. From Hölder's inequality we therefore obtain

$$
\left\|g 1_{V}\right\|_{A}:=\left\|\left(g 1_{V}\right)^{\wedge}\right\|_{1} \geqslant \frac{\left\|\left(g 1_{V}\right)^{\wedge}\right\|_{2}^{3}}{\left\|\left(g 1_{V}\right)^{\wedge}\right\|_{4}^{2}} \geqslant\left(\frac{|G|}{3}\right)^{1 / 2}\left\|\left(g 1_{V}\right)^{\wedge}\right\|_{2} \geqslant \sqrt{m / 3} .
$$

Since $m>12 M^{4}$, this is contrary to (5.1), and this proves the claim.

Applying Lemma 5.3 we obtain a subgroup $H^{\prime}$ such that

$$
\mathbb{E}_{x \in A} 1_{H^{\prime}}(x) \geqslant 2^{-2^{C M^{4}}} \text { and } \mathbb{E}_{x \in H^{\prime}} 1_{A}(x) \geqslant 2^{-C M^{4}}
$$

Since $f^{2} \geqslant 1_{A} / 4$ we get $\mathbb{E}_{x \in H^{\prime}} f^{2}(x) \geqslant 2^{-C M^{4}}$, but this does not quite imply Proposition 5.1. By Plancherel's theorem, however, we do have

$$
\left\langle\left(f \mu_{H^{\prime}}\right)^{\wedge}, \widehat{f}\right\rangle=\left\langle f \mu_{H^{\prime}}, f\right\rangle=\mathbb{E}_{x \in H^{\prime}} f^{2}(x) \geqslant 2^{-C M^{4}},
$$

which, since $\|f\|_{A} \leqslant M$, means that

$$
\left\|\left(f \mu_{H^{\prime}}\right)^{\wedge}\right\|_{\infty} \geqslant 2^{-C M^{4}} / M \geqslant 2^{-C^{\prime} M^{4}} .
$$

By the definition of the Fourier transform this yields an $r$ such that

$$
\left|\mathbb{E}_{x \in H^{\prime}} f(x)(-1)^{r^{T} x}\right| \geqslant 2^{-C M^{4}}
$$

Taking $H=H^{\prime} \cap\{r\}^{\perp}$, it is clear that

$$
\left\|\psi_{H} f\right\|_{\infty} \geqslant \sup _{x \in H^{\prime}}\left|\mathbb{E}_{y \in x+H} f(y)\right| \geqslant 2^{-C M^{4}} .
$$

Finally we note that

$$
\mathbb{E} 1_{H} \geqslant \mathbb{E} 1_{H^{\prime}} / 2 \geqslant 2^{-2^{C M^{4}}} \mathbb{E} 1_{A}
$$

and that

$$
\mathbb{E} 1_{A} \geqslant \frac{\left\|f_{\mathbb{Z}}\right\|_{1}}{\left\|f_{\mathbb{Z}}\right\|_{\infty}} \geqslant \frac{\left\|f_{\mathbb{Z}}\right\|_{1}}{\|f\|_{\infty}+\epsilon} \geqslant \frac{\left\|f_{\mathbb{Z}}\right\|_{1}}{\|f\|_{A}+\epsilon} \geqslant \frac{\left\|f_{\mathbb{Z}}\right\|_{1}}{M+\epsilon} \geqslant \frac{\left\|f_{\mathbb{Z}}\right\|_{1}}{M+1},
$$

two estimates which together imply the claimed lower bound on $\mathbb{E} 1_{H}$. 


\section{The main ARgument}

The basic strategy for proving Theorem 1.3 is that of an induction on $M$. Our first lemma provides the main inductive step. The most noteworthy feature of this lemma is that, in order to make the induction work, one cannot restrict attention to boolean functions $f: G \rightarrow\{0,1\}$. It is necessary to consider almost integer-valued functions as well.

Lemma 6.1 (Inductive step). Suppose that $f: G \rightarrow \mathbb{R}$ is $\epsilon$-almost integer-valued with $\|f\|_{A} \leqslant M$, where $\epsilon \leqslant 2^{-C M^{4}}$. Then we may decompose $f$ as $f_{1}+f_{2}$, where each $f_{i}$ is $\epsilon^{\prime}$-almost integer-valued for some $\epsilon^{\prime} \leqslant 2^{C M} \epsilon$, and for $i=1,2$ one of the following two alternatives holds:

(1) $\left(f_{i}\right)_{\mathbb{Z}}$ may be written as $\sum_{j=1}^{L} \pm 1_{H_{j}}$, where each $H_{j}$ is a subgroup of $G$ and

$$
L \leqslant 2^{2^{C M^{4}} / \epsilon}
$$

(2) $\left\|f_{i}\right\|_{A}$ is most $\|f\|_{A}-\frac{1}{2}$.

Proof. If $M \leqslant 1 / 2$ then $f_{\mathbb{Z}}=0$, and so option (1) vacuously holds. Assume, then, that $M \geqslant 1 / 2$. We begin by applying Proposition 5.1. This provides a subgroup $H \leqslant G$ such that

$$
\mathbb{E} 1_{H} \geqslant 2^{-2^{C M^{4}}}\left\|f_{\mathbb{Z}}\right\|_{1} \text { and }\left\|\psi_{H} f\right\|_{\infty} \geqslant 2^{-C M^{4}}
$$

Set

$$
\eta:=2^{-C M(1+\log M)} \epsilon
$$

for some large $C$, this choice being dictated by a later application of Proposition 3.7 .

By Lemma 3.4 we may find a subgroup $H^{\prime} \leqslant H$ with

$$
\operatorname{codim}\left(H: H^{\prime}\right) \leqslant M / \eta
$$

such that $f$ is $\eta$-spectrally supported on $H^{\prime}$. By averaging we have

$$
\left\|\psi_{H^{\prime}} f\right\|_{\infty} \geqslant\left\|\psi_{H} f\right\|_{\infty} \geqslant 2^{-C M^{4}}
$$

we also have

$$
\mathbb{E} 1_{H^{\prime}} \geqslant 2^{-M / \eta} \mathbb{E} 1_{H} \geqslant 2^{-2^{C M^{4}} / \epsilon}\left\|f_{\mathbb{Z}}\right\|_{1} .
$$

Define $f_{1}:=\psi_{H^{\prime}} f$ and $f_{2}:=f-\psi_{H^{\prime}} f$. Since $f$ is $\eta$-spectrally supported on $H^{\prime}$, it is an immediate consequence of Proposition 3.7 that both $f_{1}$ and $f_{2}$ are $\epsilon^{\prime}$-almost integervalued, for some $\epsilon^{\prime} \leqslant 2^{C M} \epsilon$.

It turns out that for $f_{2}$ alternative (2) always holds, that is to say $\left\|f_{2}\right\|_{A} \leqslant\|f\|_{A}-\frac{1}{2}$. From the Fourier definition of $\psi_{H^{\prime}}$ one sees that the supports of $\widehat{f}_{1}$ and $\widehat{f}_{2}$ are disjoint, and hence that

$$
\|f\|_{A}=\left\|f_{1}\right\|_{A}+\left\|f_{2}\right\|_{A} .
$$

Thus we need only show that $\left\|f_{1}\right\|_{A} \geqslant 1 / 2$. To see this, note that from (6.1) we have

$$
\left\|\left(f_{1}\right)_{\mathbb{Z}}\right\|_{\infty} \geqslant\left\|f_{1}\right\|_{\infty}-\epsilon^{\prime} \geqslant 2^{-C M^{4}}-\epsilon^{\prime}>0 .
$$

Since $\left(f_{1}\right)_{\mathbb{Z}}$ is integer-valued, this of course means that

$$
\left\|\left(f_{1}\right)_{\mathbb{Z}}\right\|_{\infty} \geqslant 1
$$


whence

$$
\left\|f_{1}\right\|_{A} \geqslant\left\|f_{1}\right\|_{\infty} \geqslant 1-\epsilon^{\prime} \geqslant 1 / 2 .
$$

To conclude the proof, then, we need only show that if $\left\|f_{2}\right\|_{A}<1 / 2$ then $\left(f_{1}\right)_{\mathbb{Z}}$ may be written as a \pm 1 sum of not too many cosets of $H^{\prime}$. The hypothesis on $\left\|f_{2}\right\|_{A}$ then ensures that $\left\|f_{2}\right\|_{\infty}<\epsilon^{\prime}$ which is certainly at most $1 / 10$. Also $f$ is $1 / 10$-almost integer valued so

$$
\left\|f_{\mathbb{Z}}-\psi_{H^{\prime}} f\right\|_{\infty} \leqslant 1 / 10+\left\|f-\psi_{H^{\prime}} f\right\|_{\infty} \leqslant 1 / 5 \text {. }
$$

Thus $f_{\mathbb{Z}}$ is within $1 / 5$ of a function which is constant on cosets of $H^{\prime}$. Since $f_{\mathbb{Z}}$ is integer-valued, this can only be the case if $f_{\mathbb{Z}}$ is itself constant on cosets of $H^{\prime}$, that is to say

$$
f_{\mathbb{Z}}=\sum_{j=1}^{L} c_{j} 1_{x_{j}+H^{\prime}}
$$

for some $x_{1}, \ldots, x_{L}$ which are distinct modulo $H^{\prime}$ and some non-zero integers $c_{j},\left|c_{j}\right| \leqslant$ $\left\|f_{\mathbb{Z}}\right\|_{\infty} \leqslant\|f\|_{A}+\epsilon \leqslant 2 M$.

Recall that the subgroup $H^{\prime}$ is such that $\mathbb{E} 1_{H^{\prime}} \geqslant 2^{-2^{C M^{4}} / \epsilon}\left\|f_{\mathbb{Z}}\right\|_{1}$, and since we obviously have

$$
\left\|f_{\mathbb{Z}}\right\|_{1} \geqslant L \mathbb{E} 1_{H^{\prime}}
$$

it follows immediately that $L \leqslant 2^{2^{C M^{4}} / \epsilon}$. The result follows upon noting that any coset in $G$ is either a subgroup, or else its characteristic function can be expressed as $1_{H_{1}}-1_{H_{2}}$ for two subgroups $H_{1}, H_{2} \leqslant G$.

Proof of Theorem 1.3. We apply Lemma 6.1 iteratively, starting with the observation that if $f: G \rightarrow\{0,1\}$ is a boolean function then $f$ is 0 -almost integer-valued, and hence an $\epsilon_{0}$-almost integer-valued for any $\epsilon_{0}>0$. An appropriate choice of $\epsilon_{0}$ will be made later. Split

$$
f=f_{1}+f_{2}
$$

according to Lemma 6.1, Each $f_{i}$ is $\epsilon_{1}$-almost integer-valued, where

$$
\epsilon_{1} \leqslant 2^{C M} \epsilon_{0}
$$

and is such that either $\left(f_{i}\right)_{\mathbb{Z}}$ is a sum of at most $2^{2^{C M^{4}} / \epsilon}$ functions of the form $\pm 1_{H}$ (in which case we say it is finished), or else we have $\left\|f_{i}\right\|_{A} \leqslant M-\frac{1}{2}$.

Now split any unfinished functions $f_{i}$ using Lemma 6.1 again, and so on (we will discuss the admissibility of this shortly). This procedure will result in the definition of parameters $\epsilon_{0} \leqslant \epsilon_{1} \leqslant \ldots$ satisfying

$$
\epsilon_{j+1} \leqslant 2^{C M} \epsilon_{j}
$$

for all $j$. After at most $2 M-1$ steps all functions will be either finished or will have $\|\cdot\|_{A}$-norm at most $1 / 2$, in which case they are finished for trivial reasons. Thus we have a decomposition

$$
f=\sum_{k=1}^{K} f_{k},
$$


where $K \leqslant 2^{2 M}$, each function $f_{k}$ is $\epsilon_{2 M}$-almost integer-valued and, for each $k,\left(f_{k}\right)_{\mathbb{Z}}$ may be written as a sum of at most $2^{2^{C M^{4}} / \epsilon_{0}}$ functions $\pm 1_{H}$. Now if $\epsilon_{0}$ is chosen so that $\epsilon_{2 M} \leqslant 2^{-2 M} / 5$ then

$$
\left\|f-\sum_{k=1}^{K}\left(f_{k}\right)_{\mathbb{Z}}\right\|_{\infty} \leqslant 1 / 5 .
$$

However $f$ only takes values in $\{0,1\}$ so it follows that

$$
f=\sum_{k=1}^{K}\left(f_{k}\right)_{\mathbb{Z}},
$$

which means that $f$ can be written as a sum of at most $2^{2^{C M^{4}} / \epsilon_{0}}$ functions $\pm 1_{H}$.

The condition $\epsilon_{2 M} \leqslant 2^{-2 M} / 5$ is not the strongest condition that we require on $\epsilon_{0}$. In the repeated applications of Lemma 6.1 we must ensure that $\epsilon \leqslant 2^{-C M^{4}}$ is always satisfied, and so we require

$$
\epsilon_{2 M} \leqslant 2^{-C M^{4}}
$$

In view of (6.2) it is clear, however, that we may choose $\epsilon_{0} \geqslant 2^{-C M^{4}}$, for some suitably large $C$, so that this is indeed always satisfied. This concludes the proof of Theorem 1.3 .

\section{Concluding REMARKS}

Note that our proof of Theorem 1.3 actually proves the following slightly stronger result.

Proposition 7.1. Suppose that $f: G \rightarrow \mathbb{R}$ is a function with $\|f\|_{A} \leqslant M$, and which is $\epsilon$-almost integer-valued for some $\epsilon \leqslant 2^{-C M^{4}}$. Then the function $f_{\mathbb{Z}}$ can be written as a combination of at most $2^{2^{C M^{4}}}$ functions of the form $\pm 1_{H}$.

In the same way that Theorem 1.3 can be seen as a quantitative version of Cohen's result, this proposition can be seen as a quantitative version of some results of Méla [HMP86, Mél82]. An example in Méla's work [HMP86, Mél82] shows that $\epsilon$ must be smaller than $2^{-c M}$ for such a theorem to hold.

\section{ACKNOWLEDGEMENTS}

The authors are very grateful to Ryan O'Donnell for supplying the references Bel92 and Man94.

\section{REFERENCES}

[Bel92] M. Bellare. A technique for upper bounding the spectral norm. Proceedings of the Fifth Annual Workshop on Computational Learning Theory, pages 62-70, 1992.

[Bog39] N. Bogolioùboff. Sur quelques propriétés arithmétiques des presque-périodes. Ann. Chaire Phys. Math. Kiev, 4:185-205, 1939.

[Cha02] M.-C. Chang. A polynomial bound in Freĭman's theorem. Duke Math. J., 113(3):399-419, 2002. 
[Coh60] P. J. Cohen. On a conjecture of Littlewood and idempotent measures. Amer. J. Math., 82:191-212, 1960.

[Gow98] W. T. Gowers. A new proof of Szemerédi's theorem for arithmetic progressions of length four. Geom. Funct. Anal., 8(3):529-551, 1998.

[Gre02] B. J. Green. Restriction and Kakeya phenomena. http://www.dpmms.cam.ac.uk/ bjg23, 2002.

[Gre05] B. J. Green. Finite field models in additive combinatorics. In Surveys in combinatorics 2005, volume 327 of London Math. Soc. Lecture Note Ser., pages 1-27. Cambridge Univ. Press, Cambridge, 2005.

[HMP86] B. Host, J.-F. Méla, and F. Parreau. Analyse harmonique des mesures. Astérisque, (135136):261, 1986.

[Man94] Y. Mansour. Learning Boolean functions via the Fourier transform. Theoretical Advances in Neural Computation and Learning, pages 391-424, 1994.

[Mél82] J.-F. Méla. Mesures $\varepsilon$-idempotentes de norme bornée. Studia Math., 72(2):131-149, 1982.

[Rud60] W. Rudin. Trigonometric series with gaps. J. Math. Mech., 9:203-227, 1960.

[Rud90] W. Rudin. Fourier analysis on groups. Wiley Classics Library. John Wiley \& Sons Inc., New York, 1990. Reprint of the 1962 original, A Wiley-Interscience Publication.

[Ruz96] I. Z. Ruzsa. Sums of finite sets. In Number theory (New York, 1991-1995), pages 281-293. Springer, New York, 1996.

[Ruz99] I. Z. Ruzsa. An analog of Frĕman's theorem in groups. Astérisque, (258):xv, 323-326, 1999. Structure theory of set addition.

[Sae68a] S. Saeki. On norms of idempotent measures. Proc. Amer. Math. Soc., 19:600-602, 1968.

[Sae68b] S. Saeki. On norms of idempotent measures. II. Proc. Amer. Math. Soc., 19:367-371, 1968.

[San08] T. Sanders. A note on Freŭman's theorem in vector spaces. Combin. Probab. Comput., 17(2):297-305, 2008.

[TV06] T. C. Tao and H. V. Vu. Additive combinatorics, volume 105 of Cambridge Studies in Advanced Mathematics. Cambridge University Press, Cambridge, 2006.

Department of Pure Mathematics and Mathematical Statistics, University of Cambridge, Wilberforce Road, Cambridge CB3 0WA, England

E-mail address: b.j.green@dpmms.cam.ac.uk

Department of Pure Mathematics and Mathematical Statistics, University of CamBridge, Wilberforce Road, Cambridge CB3 0WA, England

E-mail address: t.sanders@dpmms.cam.ac.uk 